NASA-TM-85967

NASA Technical Memorandum 85967

Airborne Astronomy Program

Medium Altitude Misssions Branch

Preprint Series 016

NASA-TM-85967 19840018455

(n)

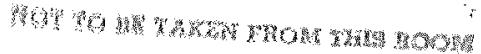

Detection of Shocked Atomic Gas in the Kleinmann-Low Nebula

M.W. Werner, M.K. Crawford,

R. Genzel, D.J. Hollenbach,

C.H. Townes, and

Dan M. Watson

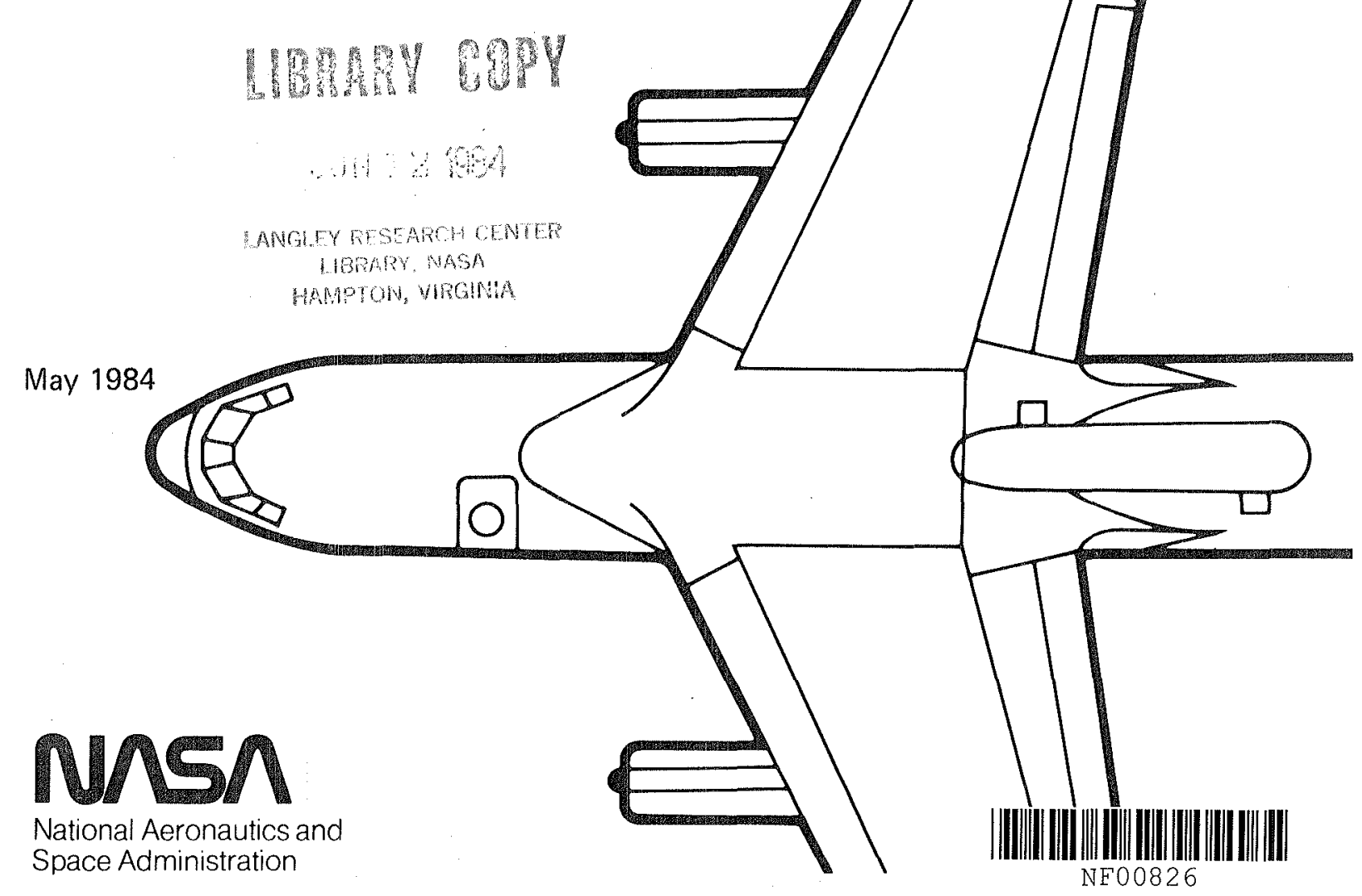




\section{Detection of Shocked Atomic Gas in the Kleinmann-Low Nebula}

M. W. Werner

D. J. Hollenbach, NASA Ames Research Center, Moffett Field, California

M. K. Crawford

R. Genzel, University of California, Berkeley, California

Dan M. Watson, California Institute of Technology, Pasadena, California 


\title{
Detection of Shocked Atomic Gas in the Kleinmann-Low Nebula
}

\author{
M.W. Werner', M.K. Crawford ${ }^{2}$, R. Genzel'2. D.J. Hollenbach'1, \\ C.H. Townes ${ }^{2}$, and Dan M. Watson
}

\begin{abstract}
We have mapped the $63 \mu \mathrm{m}{ }^{3} P_{1} \rightarrow{ }^{3} P_{2}$ fine structure line emission of neutral atomic oxygen at the center of the Orion nebula with a resolution of $30^{\prime \prime}$. There are three main emission peaks. One is associated with the region of strongest thermal radio continuum radiation close to the Trapezium cluster, and probably arises at the interface between the HII region and the dense Orion molecular cloud. The other two [OI] line emission peaks, associated with the Kleinmann-Low nebula, are similar in both distribution and velocity to those of the $2 \mu \mathrm{m} \mathrm{S}(1)$ line of molecular hydrogen and of the high-velocity wings of rotational $\mathrm{CO}$ emission. The [OI] line profiles in the $\mathrm{KL}$ region are resolved at a resolution of $45 \mathrm{~km} \mathrm{~s}^{-1}$ and have an intrinsic width of about $50 \mathrm{~km} \mathrm{~s}^{-1}$, while the line profile in the vicinity of the Trapezium is unresolved. We show that the [OI] emission from the KL nebula can be produced in the shocked gas associated with the mass outflows in this region and is an important coolant of the shocked gas.
\end{abstract}

Subject Headings: infrared: sources - infrared: spectra - nebulae: Orion nebula.

\section{Introduction}

The Kleinmann-Low (KL) nebula in Orion is the site of a violent mass outflow associated with the formation of massive stars (c.f., the review by Genzel and Downes 1982). Infrared emission lines from highly excited states of $\mathrm{H}_{2}, \mathrm{OH}$, and $\mathrm{CO}$ have been detected from this region (cf., Gautier et al. 1976: Storey et al. 1981: Beck et al. 1982); this emission is probably excited in shocks occurring where dense shells driven by the mass outtlow strike the ambient molecular cloud material. In this paper, we

INASA Ames Research Conter, Moffett Field. C. 94035

${ }^{2}$ UC Berkeley, Dept. of Physics, Berkeley, C $1.9+720$

${ }^{3}$ California Institute of Technoiggy, Pasadenit, CA. 91125 
report mapping of the central $2.5^{\prime}$ of the Orion-KL region with $30^{\prime \prime}$ resolution and show spectra taken at $45 \mathrm{~km} \mathrm{~s}^{-1}$ resolution which together reveal emission in the atomic oxygen ${ }^{3} P_{1} \rightarrow{ }^{3} P_{2}$ ground state fine structure transition associated with the $\mathrm{KL}$ nebula. This transition is expected to be the dominant cooling transition for dense $\left(10^{8} \geqslant n \geqslant 10^{3}\right)$ atomic interstellar gas for temperatures between 100 and 5000 K.

\section{Observations and Results}

\section{1) Observations}

The observations were carried out with the $91 \mathrm{~cm}$ telescope of the Kuiper Airborne Observatory in 1983 February and 1983 October, using the UC Berkeley tandem Fabry-Perot spectrometer described by Storey, Watson and Townes (1980). The beam had a FWHM of $30^{\prime \prime}$, with an effective area of a $38^{\prime \prime}$ diameter disk. In February 1983, the instrumental profile was Lorentzian with a FWHM of $170 \mathrm{~km} \mathrm{~s}^{-1}$. A chopper spacing of $4^{\prime}$ in azimuth was used (approximately east-wesi). Spectral scans were obtained at approximately forty points (typical integration time 1 minute per point) on a grid spaced by $20^{\prime \prime}$ in both right ascension and declination. Each spectral scan included the line and the adjacent continuum, so that maps of line and continuum intensity were produced simultaneously with good relative positioning. Relative positions across the map are accurate to 5". Absolute positions were obtained by assuming that the continuum peak is at R.A. $=5^{h} 32^{m} 46^{s} 8$, Dec. $=-05^{\circ} 24^{\prime} 25^{\prime \prime}(1950$, see Wynn-Williams et al. 1984). Repeated measurements of the line intensity at a point near the map center agreed $10 \pm 10 \%$, and it is this reproducibility, rather than the signal-to-noise ratio, which is the limiting uncertainty in the quality of the map presented in Figure 1. Observations were made during two flights in October, 1983, at velocity resolutions of 60 and $45 \mathrm{~km} \mathrm{~s}^{-1}$ towards several points on the map made in February; the $45 \mathrm{~km} \mathrm{~s}^{-1}$ spectra are shown in Figure 2. The beam size was again $30^{\prime \prime}$, and the chopper spacing was $5^{\prime}$ in azimuth, which was again approximately east-west. Radial velocities were calibrated by measuring the relative wavelength separation between the [OI] lines in Orion (rest wavelength $63.18372 \mu \mathrm{m})$ and an $\mathrm{H}_{2} \mathrm{O}$ line at $63.3236 \mu \mathrm{m}$. The line fluxes in Figures 1 and 2 were calibrated by measurements of the line to continuum ratio toward the continuum peak. We assumed a flux density 
of $7 \times 10^{4} \mathrm{Jy}$ for the $\mathrm{KL}$ continuum in a $30^{\prime \prime}$ beam (scaling by 0.6 the flux densities given by Werner et al. 1976). The overall calibration uncertainty is estimated to be $\pm 25 \%$.

\section{2) Spatial Distribution}

Figure 1 shows the spatial distribution of $63 \mu \mathrm{m}$ line and continuum flux over the region mapped, compared with other relevant data. The [OI] line emission shows a peak $10^{\prime \prime} \mathrm{SW}$ of the Trapezium cluster and a double-peaked ridge near the KL nebula. The peak near the Trapezium is very similar in position and shape to the $5 \mathrm{GHz}$ radio continuum emission. [OI] line emission in this region probably comes from dense $\left(n_{H} \sim 10^{4-5} \mathrm{~cm}^{-3}\right)$, warm $(T \sim a$ few hundred $K)$ gas at the interface between the HII region and the molecular cloud. This interface region will be discussed in a forthcoming paper (Ellis and Werner, 1984; see also Jaffe and Pankonin, 1978). The [OI] peaks in the KL region are close to but somewhat interior to the emission lobes of hot molecular hydrogen (Figure lb) and are also similar to the distribution of high-velocity rotational CO emission (cf. Erickson et al. 1982). The lower resolution data of Naylor et al. (1982) and Ellis and Werner (1984) also clearly show an extension of the $63 \mu \mathrm{m}$ emission from the Trapezium toward the BN-KL region but lack sufficient resolution to identify a separate peak.

\section{3) Kinematics}

Figure 2 shows [OI] spectra taken with a resolution of $45 \mathrm{~km} \mathrm{~s}^{-1}$ toward the radio peak west of the Trapezium, $\mathrm{H}_{2}$ peaks 1 and 2 and the $\mathrm{KL}$ peak (the center of the $63 \mu \mathrm{m}$ continuum emission). The line toward the radio peak is unresolved; the line shape is identical with the instrumental profile. The LSR velocity of the line is $8.5 \pm 3 \mathrm{~km} \mathrm{~s}^{-1}$ which is in good agreement with the velocity centroid of the $6300 \AA$ line of [OI] at this position (Münch and Taylor, 1974). The line profiles near the KL nebula are resolved and the lines are significantly blueshifted. The line width at $\mathrm{H}_{2}$ peak 1 is $67 \pm 5 \mathrm{~km} \mathrm{~s}^{-1}$ FWHM, and the peak of the line is at $v_{\mathrm{LSR}}=0 \pm 3 \mathrm{~km} \mathrm{~s}^{-1}$. The line widths at $\mathrm{H}_{2}$ peak 2 and the KL peak are $61 \pm 5 \mathrm{~km} \mathrm{~s}^{-1}$ and they are centered at $\mathrm{v}_{\mathrm{ISR}}=+3 \pm 3 \mathrm{~km} \mathrm{~s}^{-1}$. Most of the difference between these line profiles and the unresolved line toward the radio peak is probably due to the influence of high velocity [OI] emission, olueshifted by about 20 to $50 \mathrm{~km} \mathrm{~s}^{-1}$. The presence of strong blueshifted wings is also characteristic of the $H_{2} 2 \mu \mathrm{m}$ lines toward peaks 1 and 2 (Nadeau et al. 1982). The 
intrinsic width of the [OI] line towards the KL region may be estimated, by comparing the line width observed at $45 \mathrm{~km} \mathrm{~s}^{-1}$ with that at $60 \mathrm{~km} \mathrm{~s}^{-1}$ resolution. to be about FWHM $50 \mathrm{~km} \mathrm{~s}^{-1}$ at $\mathrm{H}_{2}$ peak 1 and $40 \mathrm{~km} \mathrm{~s}^{-1}$ toward the KL peak. These are lower limits to the width of the emission associated with the KL nebula itself since the profile probably includes a narrow component of line emission from the interface [OI] gas in front of $\mathrm{KL}$.

\section{Discussion}

\section{1) Properties of the [OI]- Emitting Gas in the KL-nebula}

The [OI]-emitting gas in the KL nebula has a large velocity dispersion and its emission has a spatial distribution similar to that of the $2 \mu \mathrm{m}$ emission from hot hydrogen. It is, therefore, likely that the $63 \mu \mathrm{m}$ [OI] emission is produced in shocks due to the mass outflow from the center of the KL nebula. The [OI] line is of substantial importance to the gas cooling in the shocked region since its luminosity is of the same order of magnitude as the luminosities of the $\mathrm{H}_{2}$ and $\mathrm{CO}$ lines. The peak surface bright-

ness of the line near $\mathrm{H}_{2}$ peak 1 is $3.3 \pm 1 \times 10^{-2} \mathrm{erg} \mathrm{s}^{-1} \mathrm{~cm}^{-2} \mathrm{ster}^{-1}$, after subtraction of a background of about $2.5 \times 10^{-2} \mathrm{erg} \mathrm{s}^{-1} \mathrm{~cm}^{-2} \mathrm{ster}^{-1}$ which may originate in the interface to the HII region. The total $63 \mu \mathrm{m}$ line luminosity from the shocked region is $50 \pm 25 \mathrm{~L}_{\odot}$, which is only about $3-4$ times smaller than the total luminosity of all $\mathrm{H}_{2}$ infrared lines $\left(-200 \mathrm{~L}_{\odot}\right.$, c.f., Scoville et al. 1982, Beckwith et al. 1983 ), or the luminosity of the far-IR CO lines $\left(150 \mathrm{~L}_{\odot}\right)$. The minimum column density of [OI] gas required to produce the observed surface brightness is $4.5 \times 10^{17} \mathrm{~cm}^{-2}$, assuming statistical equilibrium at high density $\left(\mathrm{n} \geqslant 5 \times 10^{5} \mathrm{~cm}^{-3}\right)$ and $\mathrm{T}>>230 \mathrm{~K}$. This is probably a good assumption for the parameters of the [OI] emitting gas in the KL nebula. The ratio of $63 \mu \mathrm{m}$ intensity to that of the second [OI] line at $145 \mu \mathrm{m}$ toward the center of the $\mathrm{KL}$ nebula is between 20 and 40 (Ellis and Werner, 1984). This range is consistent with optically thin emission from dense gas $\left(\mathrm{n}_{\mathrm{H}} \sim 10^{5 \pm 1} \mathrm{~cm}^{-3}\right)$ at high temperature $\left(\mathrm{T}-300\right.$ to $1500 \mathrm{~K}$ ) and is also characteristic of shocked gas. With a width of $50 \mathrm{~km} \mathrm{~s}^{-1}$, the line has negligible opacity $\left(\tau_{63}-10^{-2}\right)$. Assuming all oxygen atoms are in the form of OI and a cosmic abundance of oxygen $\left(O / 11=6.6 \times 10^{-4}\right)$, the lower limit to the column density of hydrogen nuclei in the $[\mathrm{OI}]$ emitting region is $7 \times 10^{20} \mathrm{~cm}^{-2}$, which corresponds to a mass of $\sim 0.15 \mathrm{M}_{3}$. This is 
significantly larger than the amount of very hot hydrogen gas behind the molecular shock $(T \geqslant 2000 \mathrm{~K}$ : $\mathrm{N}_{11}-5 \times 10^{19} \mathrm{~cm}^{-2}$, c.f.. Beckwith et al. 1983) but less than the hydrogen column density estimated for the medium temperature molecular gas in this region $\left(T \sim 500-1000 \mathrm{~K}: \mathrm{N}_{11}-2\right.$ to $6 \times 10^{21} \mathrm{~cm}^{-2}$, cf. Watson et al. 1984).

\section{2) [OI] Emission from the Cloud Shock}

The mass outflow in the KL nebula drives shocked, neutral gas into the ambient interstellar medium. As pointed out by Castor, McCray, and Weaver (1975), Hollenbach (1982), and Chernoff, Hollenbach, and McKee (1982, hereafter CHM), two separate shock fronts will exist under these circumstances. The "wind shock" occurs at the point where the stellar wind collides with the inner edge of the shocked, expanding material. A second shock, (the "cloud shock") occurs where the outer edge of the expanding material strikes the ambient molecular cloud. Draine and Roberge (1982) and CHM have modeled the cloud shock as a C-type shock in which the magnetic field effectively "cushions" the shock front. These models have successfully reproduced the intensities of the high excitation molecular lines of $\mathrm{H}_{2}, \mathrm{OH}$ and $\mathrm{CO}$ observed in the $\mathrm{KL}$ region. In such a shock, the [OI] $63 \mu \mathrm{m}$ line is predicted to be two or more orders of magnitude fainter than the integrated $\mathrm{H}_{2}$ emission, because any preshock atomic oxygen is rapidly converted to $\mathrm{H}_{2} \mathrm{O}$ in the hot post-shock region. By contrast, the present observations show that the $63 \mu \mathrm{m}$ line is within an order of magnitude as intense as the total $\mathrm{H}_{2}$ emission. The column density of medium temperature $(\mathrm{T}>500 \mathrm{~K}) \mathrm{C}$-shocked gas, however, is sufficient to produce the [OI] emission if $-10 \%$ of the oxygen could be maintained as [OI]. Thus, if our present understanding of the chemistry in this region is inadequate, it is possible that the [OI] emission could in fact be produced in the molecular shock.

\section{3) The Wind Shock}

Alternatively, the [OI] emission may come from the wind shock (Hollenbach. 1984; Draine and Roberge, 1982). If the outlow has a characteristic speed in excess of $100 \mathrm{~km} \mathrm{~s}^{-1}$, it strikes the inner surface of the compressed shell at a speed in excess of $50 \mathrm{~km} \mathrm{~s}$. This is sufficient to dissociate and significantly ionize the wind material, which leads to formation of a J-type wind shoch front with sharp density and temperature discontinuities and high post-shock temperatures $\left(T>10^{+} \mathrm{K}\right)$. As the gas 
behind the shock front cools and recombines. it passes through a range of temperatures from approximately 5000 to $100 \mathrm{~K}$ in which [OI] $63 \mu \mathrm{m}$ emission is the dominant coolant for the largely atomic gas. Because the $63 \mu \mathrm{m}$ line is the dominant coolant in this temperature range, about $5000 \mathrm{k}$ of energy $(\mathrm{k}=$ $\left.1.38 \times 10^{-16} \mathrm{erg} \mathrm{K}^{-1}\right)$ is radiated in the line for each nucleon which crosses the shock front. Thus the flux of material into the shocks, $\mathrm{n}_{\mathrm{H}} \mathrm{v}\left(\mathrm{cm}^{-2} \mathrm{sec}^{-1)}\right.$, is related to the surface brightness, $\mathrm{I}$, of the $63 \mu \mathrm{m}$ line by:

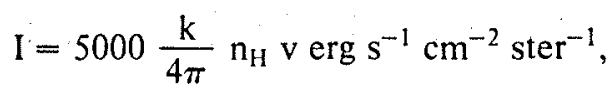

where $n_{H 1}\left[\mathrm{~cm}^{-3}\right]$ is the preshock hydrogen density in the wind, and $v$ is the relative velocity between wind and shell. Detailed calculations over the range $40 \mathrm{~km} \mathrm{~s}^{-1}<\mathrm{v}<200 \mathrm{~km} \mathrm{~s}^{-1}$ and $10 \mathrm{~cm}^{-3}<\mathrm{n}_{\mathrm{H}}$ $<10^{+5} \mathrm{~cm}^{-3}$ show that the [OI] $63 \mu \mathrm{m}$ intensity lies within a factor of two of this linear relationship for $\mathrm{n}_{11} \vee \leq 10^{12} \mathrm{~cm}^{-2} \mathrm{~s}^{-1}$ (Hollenbach, 1984). Assuming that the wind shock in the Orion-KL region falls within this parameter range, the observed [OI] $63 \mu \mathrm{m}$ intensity corresponds to a value of $n_{H} v=(6 \pm$ 2) $\times 10^{11} \mathrm{~cm}^{-2} \mathrm{~s}^{-1}$. In this limit, the total luminosity of the $63 \mu \mathrm{m}$ line gives a direct estimate of the mass loss rate $\dot{M}$ from the central object and is about $7 \mathrm{~L}_{\odot}$ if $\dot{\mathrm{M}}=10^{-3} \mathrm{M}_{\odot} \mathrm{y}^{-1}$. For the observed total [OI] luminosity of $50 \pm 25 \mathrm{~L}_{\odot}$, the corresponding mass loss rate is $(7 \pm 3.5) \times 10^{-3} \mathrm{M}_{\odot} \mathrm{y}^{-1}$, which is comparable with or somewhat higher than other estimates of the mass loss rate in the KL region. If the [OI] emission arises predominantly in the wind shock, while the $\mathrm{H}_{2}$ and $\mathrm{CO}$ infrared emission lines arise in the molecular shock, differences in the details of the line profiles and spatial distribution are expected. The $[\mathrm{OI}]$ data presented above are consistent with these expectations but do not exclude the possibility that the [OI] emission comes from the cloud shock. The [OI] $63 \mu \mathrm{m}$ emission line may be one of the few direct observational probes of $\mathrm{J}$-type wind shocks in embedded infrared sources. If [OI] emission from the wind shock can be unambiguously identified. its intensity may be an important measure for determining mass loss rates in outflow sources. 


\section{Acknowledgements:}

We thank the staff of the Kuiper Airborne Observatory for their excellent support. We are grateful to J.B. Lugten and H.B. Ellis for help with the observations and data reduction. This work was supported by NASA grant NAG2-208 (UCB) and by the Astrophysics Division of NASA Ames.

\section{References}

Beck, S.C., Bloemhof, E.E., Serabyn, E., Townes, C.H., Tokunaga, A.T., Lacy, J.H., and Smith, H.A., 1982, Ap. J. (Letters), 253, L83.

Beckwith, S., Evans, N.J., Gatley, I., Gull, G., and Russell, R.W., 1983, Ap. J., 264, 152.

Castor, J.M., McCray, R., and Weaver, R., 1975, Ap. J. (Letters), 200, L207.

Chernoff, D.F., Hollenbach, D.J., and McKee, C.F., 1982, Ap. J. (Letters), 259, L97.

Draine, B.T., and Roberge, W.G., 1982, Ap. J. (Letters), 259, L91.

Ellis, H.B., and Werner, M.W., 1984, in preparation.

Erickson, N.R., Goldsmith, P.F., Snell, R.L., Berson, R.L., Huguenin, G.R., Ulich, B.L., and Lada, C.J., 1982, Ap. J. (Letters), 261, L103.

Gautier. T.N., Fink, U., Treffers, R.R., and Larson, H.P., 1976, Ap. J. (Letters), 207, L129.

Gautier. T.N., 1979, Ph.D. Thesis, University of Arizona.

Genzel, R., and Downes, D., 1982, Regions of Recent Star Formation, ed. R.S. Roger and P.E. Dewdney (Dordrecht: Reidel), p.251.

Hollenbach, D.J., 1982, Amm. N.Y. Acad. Sc\%, 395, 242.

Hollenbach, D.J., 1984, in Proceedings of the Protostars and Planets Simposium, to be published in Icarus. Jaffe, D.T., and Pankonin. V., 1978, Ap. J., 226, 869.

Johnston, K.J., Palmer, P., Wilson, T.L., and Bieging, J.H., 1983, Ap. J. (Letters). 271, L89. 
Münch, G., and Taylor, K., 1974, Ap. J. (Letters) 192, L93.

Nadeau, D., Geballe, T.R., and Neugebauer, G., 1982, Ap. J., 253, 154.

Naylor, D.A., Emery, R., Fitton, B., Furniss, I., Jennings, R.E., and King, K.J., 1982, in Regions of Recent Star Formation, ed. R.S. Roger and P.E. Dewdney, (Dordrecht: Reidel), 73.

Scoville, N.Z., Hall, D.N.B., Kleinmann, S.G., and Ridgway, S.T., 1982, Ap. J., 253, 136.

Storey, J.W.V., Watson, D.M., and Townes, C.H., 1980, Int. J. IR and MM Waves, 1, 15.

Storey, J.W.V., Watson, D.M., Townes, C.H., Haller, E.E., and Hansen, W.L., 1981, Ap. J. (Letters). 248, L109.

Werner, M.W., Gatley, I., Harper, D.A., Becklin, E.E., Loewenstein, R.F., Telesco, C.M., and Thronson, H.A., 1976, Ap. J., 204, 420.

Wynn-Williams, C.G., Genzel, R., Becklin, E.E., and Downes, D., 1984, Ap. J., in press. 


\section{Figure Captions}

Figure 1a (left): Contours of integrated $63 \mu \mathrm{m}$ [OI] line emission toward the Orion-KL region with a resolution of $30^{\prime \prime}$ (heavy lines). Contour units are $2.5 \times 10^{-17} \mathrm{~W} \mathrm{~cm}^{-2}$ $\left(9.4 \times 10^{-3} \mathrm{erg} \mathrm{s}^{-1} \mathrm{~cm}^{-2} \mathrm{ster}^{-1}\right)$. The dashed lines are contours of $5 \mathrm{GHz}$ radio continuum emission at a resolution of 28 " (from Johnston et al. 1983, contour unit 55 mJy per beam). The crosses mark the positions of the $63 \mu \mathrm{m}$ continuum peak (the KL-nebula, upper) and of $\Theta^{\mathrm{l} C}$ Ori (lower), the brightest star in the Trapezium cluster.

Figure 1b (middle): Contours of integrated [OI] emission (heavy lines), superposed on contours of $v$ $=1 \rightarrow 0 \quad\left(\mathrm{~S}(1)\right.$ emission from $\mathrm{H}_{2}$ at $2 \mu \mathrm{m}$ (Gautier 1979), 30" beam, contour unit $6 \times 10^{-4} \mathrm{erg} \mathrm{s}^{-1} \mathrm{~cm}^{-2} \mathrm{ster}^{-1}$ ). The northwestern, stronger peak is $\mathrm{H}_{2}$ peak 1 , the southeastern peak is $\mathrm{H}_{2}$ peak 2 .

Figure 1c (right): Contours of $63 \mu \mathrm{m}$ continuum emission (heavy contours $1,2,4,6,8,10,12,14,16$, 18 times $3.8 \times 10^{3} \mathrm{Jy}$ or $1.6 \times 10^{-17} \mathrm{~W} \mathrm{~cm}^{-2}$ ), superposed on the $5 \mathrm{GHz}$ radio continuum map.

Figure 2: Spectra of $63 \mu \mathrm{m}$ [OI] emission toward several positions in the Orion-Nebula region: $10^{\prime \prime}$ west of Trapezium (bottom), $\mathrm{H}_{2}$ peak 2 (second from bottom), 63 $\mu \mathrm{m}$ continuum peak (third from bottom) and $\mathrm{H}_{2}$ peak 1 (top). Beam size is $30^{\prime \prime}$ and the instrumental profile is a Lorentzian of FWHM $45 \mathrm{~km}$ $\mathrm{s}^{-1}$ (indicated by thin lines in the upper three spectra). The bottom line is unresolved. The curvature in the continuum on the left and right of the [OI] line is due to telluric $\mathrm{H}_{2} \mathrm{O}$ absorption. The vertical scale is given separately on the $y$-axis for each spectrum. Typical integration times are 6 minutes per spectrum.

\section{Author's Addresses}

M.W. Werner and D.J. Hollenbach

NASA Ames Research Center

Moffett Field, CA. 94035

M.K. Crawtord, R. Genzel, and C.IH. Townes 
University of California, Dept. of Physics,

Berkeley, CA. 94720

Dan M. Watson

Downs Laboratory of Physics

California Institute of Technology

Pasadena, CA. 91125 


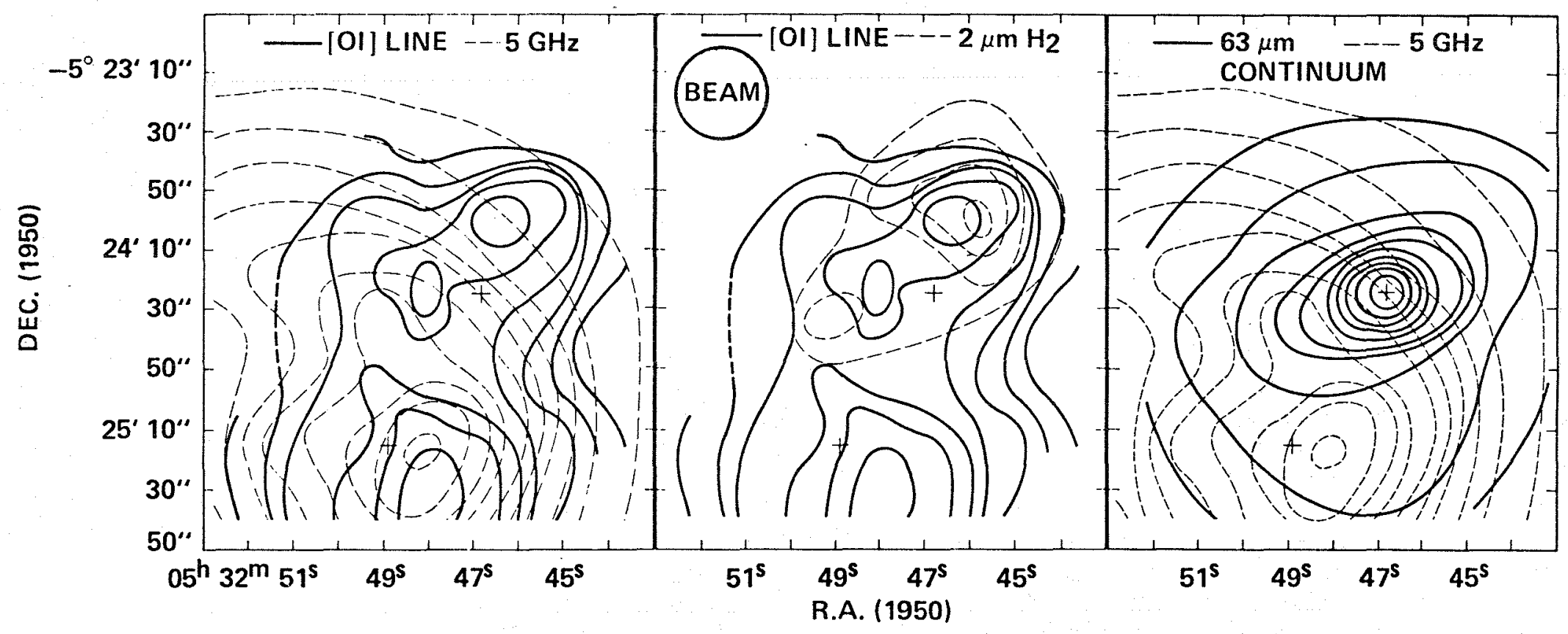

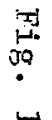




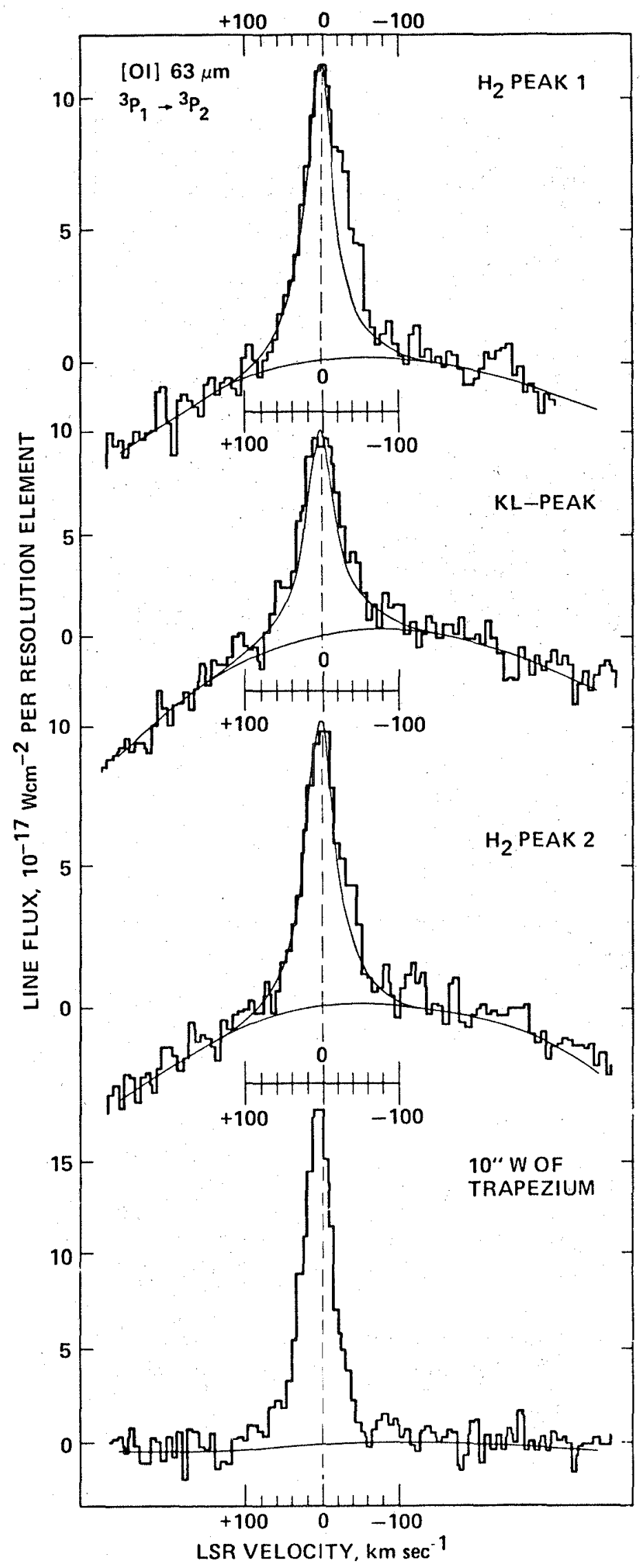

Fig. 2 


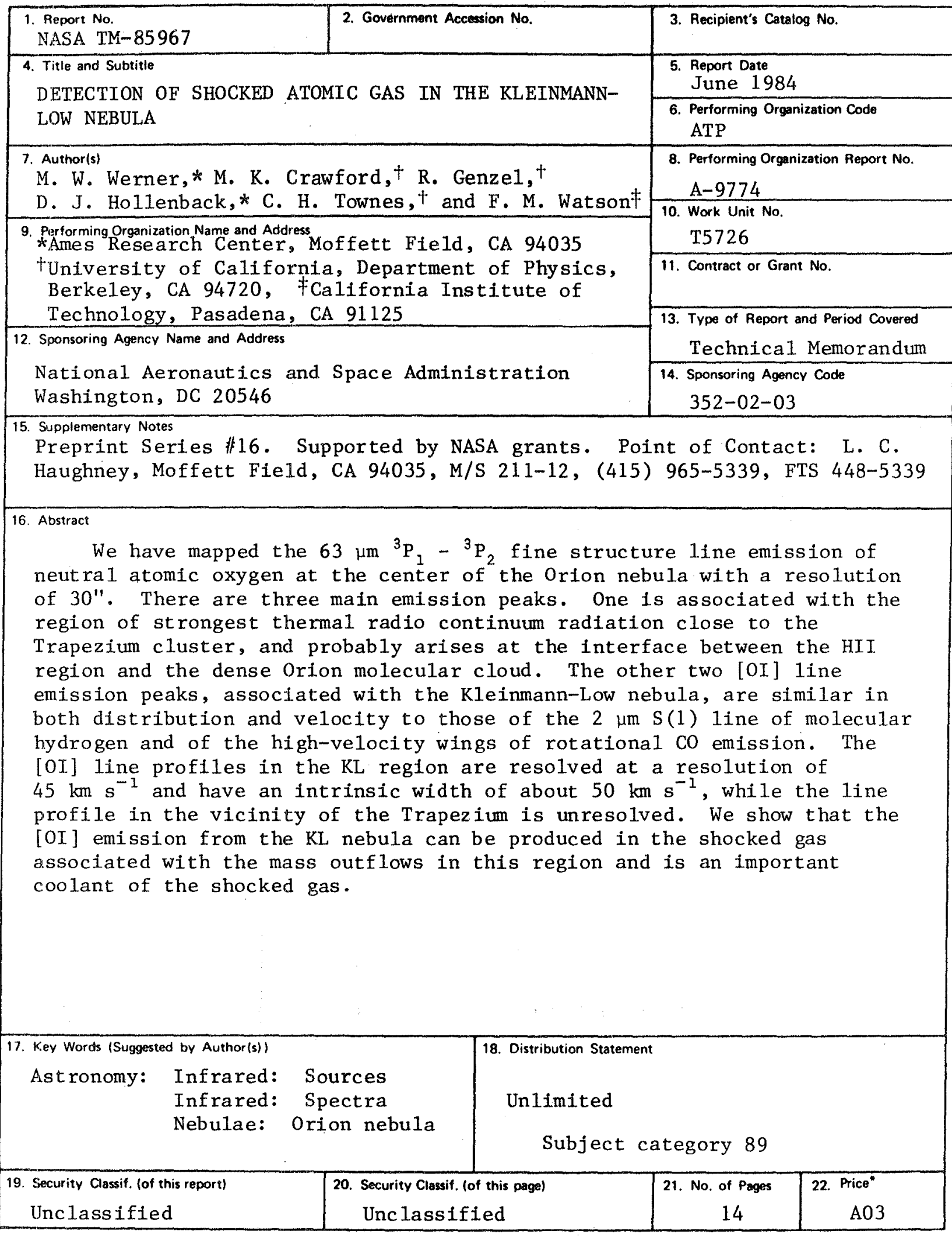

"For sale by the National Technical Information Service, Springfield, Virginia 22161 


\section{End of Document}

